\title{
Liste d'attesa e possibili soluzioni concrete
}

\author{
Gian Battista Mannone', Giuseppe Quintaliani² \\ ${ }^{1}$ Coordinatore Governo Clinico Società Italiana di Nefrologia, Roma \\ ${ }^{2}$ Blogger sanitario, Perugia
}

\begin{abstract}
Waiting list for patients. What can we do?
More and more frequently we talk about waiting lists. The interesting thing is that almost always we speak about diagnostic "tests" and almost never about visits. The lengthening of waiting lists for diagnostic services reveals a problem related not so much to medicine, but to the not regulated request of exams, sometimes useful, but almost always useless, to diagnose suspected but not clear diseases. It should alarm the medical class. Unfortunately the clinicians are not able to recognize and diagnose diseases often obvious with clear epidemiology, a problem which induces overdiagnosis and more diagnostic tests.
\end{abstract}

Keywords: Waiting list, Health care

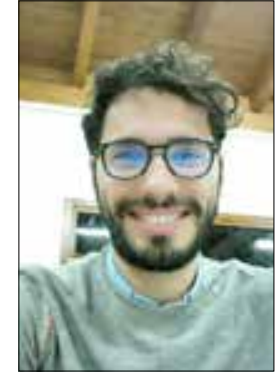

Gian Battista Mannone
Sempre più frequentemente si parla di liste d'attesa. E non c'è giorno che sulla stampa non vengano pubblicate grida di dolore per esami rimandati di mesi o anni. La cosa interessante è che quasi sempre si parla di "esami" diagnostici e quasi mai di visite. Questo dato di fatto dovrebbe preoccupare e allarmare chi prende le decisioni in politica. Infatti, l'allungamento delle liste d'attesa per prestazioni diagnostiche svela un problema legato non tanto alla medicina, ma alla richiesta non governata di esami, alle volte utili, ma, spesso, anche inutili, per diagnosticare patologie sospette ma non chiare. E dovrebbe allarmare anche la classe medica che si trova a non diagnosticare patologie spesso ovvie, con epidemiologie chiare ma che il clinico non è in grado di riconoscere, un problema a cui si cerca di porre rimedio proponendo esami diagnostici.

È noto che l'allungamento delle liste d'attesa sia uno dei diversi strumenti manageriali di razionamento delle opzioni in sanità: spesso si cela il tentativo di spostare verso il privato molte attività (1) che sicuramente il privato sa "gestire" me-

Accepted: July 12, 2016

Published online: August 1, 2016

Indirizzo per la corrispondenza:

Prof. Giuseppe Quintaliani

Via Maturanzio 31

06124 Perugia

g.quintaliani@yahoo.it glio, ma siamo sicuri che sappia farle meglio? Ci si potrebbe chiedere se professionisti che hanno in cura ricoverati debbano limitare il loro tempo di cura per fare visite agli esterni e se, quindi, il modello "privato per esterni e ospedali per interni" possa essere il più adatto, ma non è questo ora il punto (magari ci potremo ritornare). II focus è che il problema delle liste d'attesa non si affronta con l'aumento dell'offerta! L'aumento della richiesta, che, in un mercato libero, governa l'offerta, nel campo della sanità (che non è un mercato libero ma vincolato) deve essere gestito e governato rendendo la richiesta oculata e appropriata. II movimento di pensiero "from volume to value" (2) è un esempio illuminante. Come? Ci sono alcuni modi fondamentali che partono dalla consapevolezza che molte indagini sono assolutamente inutili. In questa intervista al Dr. Nino Cartabellotta, che si rifà al programma "Choosing Wisely" del Gimbe (3), si cerca di individuare, appunto, le cose inutili. E le cose inutili dovrebbero essere discusse con i MMG, con le amministrazioni e con i professionisti, chiamati a offrire prestazioni, e non essere imposte da amministrativi. Una soluzione o un tentativo di affrontare il problema potrebbe risiedere nel resoconto CUP; i professionisti che fanno gli esami potrebbero segnalare positivo/ negativo, in modo da permettere una rilevazione statistica: quanti esami vengono richiesti, quanti di essi sono positivi o negativi? In questo modo avremmo innanzitutto una rilevazione statistica dei bisogni e delle patologie da prendere in considerazione. Se, poi, gli esami fossero in maggioranza negativi, si potrebbe intervenire con progetti di formazione/ informazione. In un recente articolo (4), i radiologi hanno ammesso che il 50\% degli esami strumentali di immagine è del tutto inutile: allora, perché aumentare le ore di apertura? Per fare ancora più esami negativi e, quindi, dannosi, vista la dose 
irradiante somministrata?

Rincorrere lo screening è sacrosanto, ma solo nei pochissi$\mathrm{mi}$ casi in cui sia indicato e sono veramente pochi.

Se interessasse veramente la salute della popolazione, si applicherebbero i decreti ministeriali come il recentissimo decreto sulla cronicità (5) o, per quello che riguarda la nefrologia, il decreto sulla malattia renale cronica (6). Eppure, situazioni così ben codificate ma con lunghe liste d'attesa (in nefrologia ci sono sei mesi d'attesa per il follow-up) non vengono affrontate e si preferisce aumentare l'offerta della diagnostica. Perché si valuta solo la prima visita quando il problema è la cronicità? Bisognerebbe valutare la presa in carico e le visite successive che assicurano la costanza della cura e la prevenzione delle complicazioni. Strano, no? Senza contare che, come scrive Remuzzi su Lancet, la malattia renale cronica è la quinta causa di morbilità (7) in Italia, ma non si attuano misure di prevenzione, si smantellano le guardie attive 24 ore su 24 , non si assume personale e si lasciano pazienti cronici senza paracadute, riducendo addirittura gli accessi durante l'estate.

Un'altra soluzione potrebbe consistere nello spostare gli amministrativi nei reparti. Una recente indagine dimostrava che gli amministrativi sono tanti negli ospedali (8) e in aumento, ma molti dimenticano di dire che gli amministrativi stanno in aree a loro riservate e non nelle degenze, né tantomeno negli ambulatori! Gli amministrativi, se spostati negli ambulatori e nelle degenze, potrebbero aprire le cartelle e archiviarle, fare fotocopie, scrivere le lettere che il medico potrebbe dettare al registratore (come fanno i radiologi), sistemare le cartelle in maniera cronologica e fare tante altre piccole cose che renderebbero molto più veloci e proficue le visite. Tanto per fare un esempio: le cartelle contengono fogli perforati per permettere di agganciarli e mantenerli ordinati eppure bastano un foglio in più per la terapia, una semplice lettera di dimissione da dare al medico o altri documenti da inserire come risposte gli esami, per costringere un medico ad aprire tutti gli anelli, sfogliare i fogli uno a uno e rimetterli al loro posto in maniera ordinata. Quanto tempo viene impiegato per queste pratiche non del tutto medico-sanitarie? Invece gli amministrativi vengono mantenuti in aree a loro riservate e pochissimi di loro sono nei reparti e nessuno negli ambulatori.

Ancora: I'uso dell'informatica. Quante volte si scrive la terapia di un paziente in ambulatorio? La terapia nel computer del medico di medicina generale spesso NON viene comunicata allo specialista che, visto il grande problema nelle malattie croniche, di volta in volta può essere il cardiologo, il diabetologo, il nefrologo, il chirurgo vascolare e così via. $\mathrm{A}$ causa di questa mancanza di comunicazione, spesso si hanno $5,6,7$ terapie scritte in ognuna delle cartelle degli ambulatori dove il paziente accede e quasi sempre nessuna è congruente con le altre, ammesso che il paziente porti con sé i bugiardini delle medicine per capire effettivamente quelle che sta assumendo. Si pensi al tempo sprecato e quanto se ne potrebbe guadagnare semplicemente se la terapia del paziente fosse univoca e facilmente consultabile e consigliabile da tutti coloro che hanno in cura il paziente stesso (nel mondo normale questo si chiama condivisione dell'informazione o "sharing in cloud", un po' come succede, per esempio, con Facebook!). Non parliamo, poi, degli altri usi (anzi del non uso) dell'informatica in sanità, perché la cosa ci porterebbe molto lontano.

$E$, ancora, visto che i pazienti, per la maggior parte, sono cronici, che senso ha mandarli una volta in una sede e, poi, in un'altra e, ancora, in un'altra per poi tornare alla prima, come se fossero pacchi? La continuità della cura si perde, il paziente è frastornato e lo stesso vale anche per i medici. Non sarebbe meglio, dopo una prima visita, far collaborare, anche per via informatica (9), il MMG e lo specialista? Così, il tempo sarebbe ben speso, con grandi ritorni in tema di salute come il QOF inglese ben dimostra. $O$ perché non organizzare dei percorsi adatti per garantire la presa in carico e la salute dei pazienti?

Certo, il pallino passerebbe dai manager ai clinici e dalle amministrazioni all'epidemiologia, ma è così che funziona in tutto il mondo! In Gran Bretagna, per esempio, nessuno va a fare gli esami, neanche privati, se non li ha prescritti il MMG, il quale ci pensa mille volte a richiederli se non sono necessari. Infatti, ogni medico viene monitorato per i numeri delle prestazioni, ma, soprattutto, per i risultati: da questo dipende il suo salario. Insomma, si deve arrivare alle valutazioni e le valutazioni le fanno i clinici, non gli amministrativi! Per esempio, i criteri di accreditamento regionali spesso non prevedono la valutazione dei risultati ma solo dei volumi di attività, contraddicendo una letteratura abbondante e circostanziata. "Fare di più non significa fare meglio»: questa è la campagna varata in Italia da Slow Medicine (10). L'epidemiologia ha un grande ruolo, ora si parla di "geo-localizzazione" delle malattie (l'ha fatto anche la Lombardia) e, quindi, si dovrebbe sapere a cosa serve e dove serve. La medicina di iniziativa, inoltre, potrebbe permettere ai MMG di chiamare i pazienti secondo protocolli testati e riconosciuti. In altre parole, se io ho 100 diabetici che devono fare la visita 12 volte all'anno, dovrò assicurare 1200 prestazioni, se le prestazioni sono, invece, 5000 all'anno c'è qualcosa che non torna.

C'è anche il grande problema degli esami che portano ad altri esami, perché il miglioramento dell'accuratezza delle apparecchiature consente di aumentarne la sensibilità e la capacità di cogliere piccole alterazioni, anche di scarso rilievo; così, il sistema si complica ancora di più (11), con il ricorso a ulteriori esami (come, appunto, succede con le risonanze e la TAC). Quante volte un esame diagnostico finisce dicendo "utile controllo a 4 o 6 o 8 o 12 mesi"? La soluzione non è avere più prestazioni ma più esperti che aiutino nella clinica e decidano. Una volta, per esempio, su un giornale umbro è stata riportata la notizia che "lo screening gratuito eseguito nel corso della "Giornata della Tiroide" ha rivelato la presenza di una qualche patologia tiroidea nei $2 / 3$ (il 66\%!) della popolazione esaminata. La notizia è stata data, con molta soddisfa- 
zione, dai vertici sanitari! Forse vogliono dirci che i due terzi della popolazione hanno un cancro della tiroide? E quanti sono i decessi dovuti a questa nuova peste? Sempre gli stessi da anni. Riteniamo che, forse, il risultato andrebbe discusso con esperti perché sembra impossibile e contrario a tutta l'epidemiologia e a tutta la letteratura. La questione non è di fare di tutti gli screenati dei malati, ma selezionare quelli veri!

Anche per gli esami oncologici, che così tanto colpiscono la popolazione, il problema è lo stesso (12). Il punto è valutare sempre il costo/beneficio (in termini di salute, non di denaro!) di qualsiasi procedura (per esempio, la Svizzera non effettua più screening di $\mathrm{K}$ della mammella per i problemi riscontrati, anche se questo, a mio parere, è contro le Linee Guida internazionali). La cosa è molto dibattuta anche sulla stampa, sia non medica (13) sia scientifica (14), ma una cosa è certa: l'aumento di molti esami e indagini diagnostiche non ha portato a una riduzione della mortalità (15) e su questo i giornali seri di medicina tornano (16) sempre più spesso. Sempre più spesso si evidenzia che più aumenta l'offerta più si induce domanda! La domanda dovrebbe essere calmierata con sistemi adatti di audit professionali che valutino le aree a maggiore inappropriatezza, con campagne di stampa volte a sottolineare che la medicina non può risolvere tutti i mali e che è impossibile diagnosticare sempre e comunque un qualsiasi disturbo o doloretto. È un problema di mentalità e di organizzazione e, sicuramente, aprire la sera e la domenica per fare indagini diagnostiche non è un buon segnale di appropriatezza. Lo ripetiamo, se vogliamo fare una medicina corretta ci vogliono medici competenti a tutti i livelli, meno diagnostica e più percorsi per la malattia cronica. Le malattie croniche sono il vero flagello del nuovo secolo e i dati provenienti dalla regione Veneto mostrano come il $20 \%$ della popolazione assorba quasi il $70 \%$ delle risorse. Quindi, meno esami e più percorsi.

0 , forse, tutto è in funzione delle statistiche per apparire e non per essere? Ma tutti, un giorno, staremo male e tutti saremo dei vecchietti cronici con tante malattie. II piano cronicità indica che circa il 50\% della popolazione sopra i 65 anni ha due malattie croniche (il $68 \%$ della popolazione sopra i 75 anni): perché, allora, impegniamo risorse nella diagnostica invece che nella presa in carico? Non sarebbe preferibile, invece che cercare di apparire, trattare meglio le cronicità? II ruolo della politica è di prendere decisioni magari impopolari, ma giuste e corrette, e non di andare dietro alla demagogia! II ruolo dei tecnici, a loro volta, non è quello di assecondare la politica, ma di far riflettere con numeri, dati e scienza per poter prendere la decisione più appropriata.

In un cartello appeso nella sala riunioni di una grande multinazionale americana c'era scritto: "Without data you are just another person with an opinion".

Ovviamente, nessuno vuole dire che gli esami diagnostici non servano, ma dobbiamo usarli con oculatezza (17) e appropriatezza, con la consapevolezza che, aumentando l'offerta, si ha comunque un aumento ulteriore degli esami e non una loro riduzione. Una cosa è certa: solo lavorando con l'epidemiologia e con l'appropriatezza clinica (ben diversa da quella amministrativa di DRG, ICD9 e codici tra ambulatorio e day hospital e ticket vari) e coinvolgendo le società scientifiche, potremmo non dico risolvere, ma, almeno, arginare il problema. Una breccia in un muro si risolve riparandola, non allargandola.

\section{Liste d'attesa e attività libero-professionale}

Il problema è vecchio e si ripropone periodicamente. Silvio Garattini, dell'Istituto Mario Negri, si è espresso a favore dell'abolizione dell'intramoenia (18). Non si sono fatte attendere certo le risposte, soprattutto dei sindacati (19). Recentemente, anche il presidente della Regione Toscana Enrico Rossi ha lanciato i suoi strali in un post su Facebook (20), suscitando, di nuovo, innumerevoli proteste e prese di posizione. lo credo che, in effetti, a fronte di 11 milioni di persone che non si curano o perché non abbienti o per le liste d'attesa, tale glorioso e giusto istituto debba essere in qualche modo rivisto. Anche il Ministro Lorenzin ha espresso un suo parere, favorevole non certo all'abolizione ma alla modifica (21).

Ma è anche giusto quello che dice Rossi: I'intramoenia così è indegna ed è fonte di sperequazioni, prima di tutto tra medici che possono permettersi tale attività per le specializzazioni che più di altre possono indurre prestazioni ambulatoriali. Ma, soprattutto, nei confronti dei pazienti.

Non è certo degno di un paese civile mortificare i poveri che aspettano mesi per avere una prestazione dagli stessi medici che, a pagamento, la assicurano a chi può permetterselo.

Basta frequentare i social per capire che, come dice Rossi, aspettare mesi e sapere di poter avere un appuntamento entro due o tre giorni a pagamento induce proteste, svilimento e reazioni furiose in coloro che incappano in tale perverso meccanismo (22).

Ancora un'altra stortura: spesso abbiamo professionisti bravi a cui e' pero' difficile, se non impossibile, accedere con la normale prenoatzione CUP. Perché privare la popolazione del loro prezioso contributo? 0 , ancora, perché molti responsabili o medici limitano gli accessi all'ambulatorio istituzionale mantenendo, però, attivo quello libero-professionale, addirittura in extramoenia (23)?

Recentemente, due articoli pubblicati su riviste di alto livello internazionale puntavano l'indice sull'attività liberoprofessionale, ritenendola non etica e fonte di disuguaglianze inaccettabili $(24,25)$.

Detto questo, e proprio per non essere equivocato, io non sono contro l'attività libero-professionale, anzi potrebbe essere uno dei parametri per valutare la "clinical competence" e l'empatia dei medici. E il discorso che faccio riguarda solo ed essenzialmente l'attività privata in commistione con quella pubblica e, cioè, l'attività libero-professionale intramoenia. Noi siamo CONTRO la penalizzazione dei poveri! 


\section{Quale potrebbe essere la soluzione?}

La soluzione è la libera professione "comperata" dall'Azienda Ospedale per la quale si lavora. Semplice, no?

Tra l'altro, è un istituto contrattuale (articolo 50 del CCNL) e mi meraviglia che i sindacati si siano subito scagliati contro la proposta, inducendo nella popolazione l'idea che molti hanno della classe medica, cioè di una casta! Se fossero stati $\mathrm{i}$ sindacati ad anticipare i tempi, se si mettessero per una volta dalla parte del cittadino, non sarebbero i politici dell'una o dell'altra parte, spesso incompetenti, a fare proposte. Ormai sembra che solo Gimbe si scagli sempre e comunque per salvare il SSN. Per esempio, un intervento su doctor33 del 7 Aprile 2014 propone: «Né libera professione intramoenia né straordinario con apertura degli ambulatori fino a notte come in Veneto, ma una terza via, prevista dai contratti dei medici ospedalieri e del comparto, quella sperimentata a La Spezia da medici e infermieri del reparto di chirurgia vascolare del Sant'Andrea: i sanitari effettuano visite ed eco-Doppler come prestazioni aggiuntive fuori orario di lavoro per ridurre le liste d'attesa, con qualche risparmio per la sanità pubblica... a prezzi concordati e calmierati».

In ogni caso, l'attività libero-professionale acquistata dalle aziende sanitarie permetterebbe di avere diversi vantaggi:

1. incentiverebbe i direttori generali a utilizzare meglio le risorse interne omogeneizzando le piante organiche, governando le liste d'attesa, ricorrendo all'appropriatezza e usando il governo clinico invece di sistemi "fiduciari";

2. premierebbe i migliori, visto che un sanitario molto richiesto (e magari poco valorizzato in azienda) avrebbe carte da giocare in senso meritocratico. È ovvio che, a breve scadenza, potrebbero intervenire sistemi di marketing, ma, a lunga scadenza, un sanitario che si facesse forza solo dell'immagine, ma di scarsa qualità, sarebbe pian piano emarginato;

3. ci sarebbe un giusto equilibrio tra attività per CUP e attività acquistata, in modo da bilanciare abusi e convenienze (10 visite in 5 ore al CUP non potrebbero essere paragonate a 50 visite in 10 ore dichiarate in intramoenia);

4. limiterebbe le attività al di fuori delle mura ospedaliere facendo rientrare nella sanità pubblica molte attività private esterne;

5. permetterebbe di avere un piccolo tripAdvisor non tanto delle strutture ma dei sanitari afferenti e sarebbe interessante vedere se e quanto i giudizi dei primari o delle direzioni siano allineati con quelli dei pazienti e anche, perché no, se i giudizi sui primari effettuati dalle aziende al momento delle nomine rispecchino veramente e totalmente la realtà delle cose. Oltre, ovviamente, a permettere di bypassare le incomprensioni e le lotte di potere all'interno dei reparti. Immaginate un medico a cui tutti si vogliono affidare che, invece, sia negletto e l'ultimo nella considerazione aziendale;
6. permetterebbe di valutare le strutture che sono particolarmente sottodimensionate e dove la programmazione locale o regionale ha fallito; un sistema di controllo anche politico, dunque;

7. permetterebbe di valutare le direzioni generali sull'organizzazione delle strutture e la Corte dei Conti potrebbe essere chiamata a esprimere giudizi su quanto speso per acquistare intramoenia e su sprechi e corruzioni che sono la palla al piede della sanità. In altre parole se una direzione acquistasse intramoenia in maniera spropositata ed inappropriata, accertata con modelli di benchmarking, potrebbe essere sanzionata;

8. permetterebbe ai sanitari di guadagnare un giusto denaro al servizio dei pazienti senza essere tacciati di opportunismo;

9. permetterebbe di ridurre, ove presente, l'eccessivo ricorso ai convenzionati esterni.

Mi immagino le critiche a questa proposta ma faccio notare che pian piano si arriverà a questo. Nella stessa Toscana, il ricorso a strutture esterne convenzionate sta uccidendo la professione medica dei professionisti che ancora vogliono lavorare per il pubblico e rimanere nel pubblico. Inoltre, attenzione alle 11 ore di riposo: l'azienda sanitaria potrebbe pretendere che il sanitario si astenga da qualunque prestazione sanitaria per 11 ore, anche dalle prestazioni private, per avere, appunto, un sanitario riposato. Ancora non ci hanno pensato, ma vedrete che si arriverà anche a questo.

Così, anche attività che non consentono visite ambulatoriali potrebbero giovarsene (per esempio, la dialisi). Al paziente, anche esente, potrebbe essere richiesto il ticket, ma il sistema sanitario potrebbe avere sempre sotto controllo le prestazioni e il tipo di prestazioni, potendo, poi, formulare statistiche e previsioni di tipo epidemiologico. Le molte visite diabetologiche indicano che il diabete è un problema e potrebbe meritare un'attenzione particolare o un aumento delle strutture istituzionali. Ciò sarebbe un bel problema per i direttori che non abbiano adeguatamente e correttamente fatto una buona organizzazione delle cure.

La mia proposta non è, quindi, contro l'attività liberoprofessionale, non sono un talebano. Capisco, apprezzo e stimo i colleghi che hanno fatto questa scelta, che considero normale e giusta. Ma vorrei non penalizzare i meno abbienti, e vorrei mettere davanti alle proprie responsabilità politici, amministratori, MMG ed equipe mediche. Inoltre, ripeto, le implicazioni in termini di informazioni che tale sistema potrebbe dare sono enormi. Quindi, non abbandonerei la libera professione ma la supererei per migliorare l'assistenza e per valutare i medici più capaci, volenterosi e preparati. La libera professione è una risorsa importante nelle mani della classe medica, che deve essere valorizzata e non svilita, come un sistema salta code, e deve essere considerata come è: un riconoscimento giusto e prezioso per coloro che, nel libero mercato della sanità, sono in grado di affermare la loro pro- 
fessionalità. Ricordiamoci sempre che, in una società dove si può scegliere tutto, dal tipo di rapporto sentimentale, alla casa e al matrimonio, non è possibile scegliere il medico che ci cura nelle strutture pubbliche. La libera professione come la intendo io sarebbe un giusto riconoscimento per i bravi medici e una liberazione per i pazienti, che potrebbero scegliere un medico di fiducia. Cosa succederebbe se i pazienti scegliessero solo un medico tra 5, 6 o più in organico? Lascio a voi la risposta, ma non credo che ci sia molto da immaginarsi e sarebbe tutto a favore dei pazienti.

Le prese di posizione dei sindacati lasciano la popolazione basita di fronte a interminabili liste d'attesa. È ora che i medici siano dalla stessa parte dei pazienti, soprattutto dei più umili e meno abbienti (26). Quanto proposto dovrebbe diventare un sistema di valutazione delle capacità organizzative e del tipo di prestazioni che il pubblico è in grado di offrire. Se lo sbaglio è di tipo organizzativo, non si è tenuto in conto della epidemiologia e delle necessità della popolazione di un certo distretto, dovrebbero essere le strutture pubbliche a comprare attività libero professionale e non scaricare tutto sui pazienti. E ci dovrebbero essere rapporti stretti tra l'attività libero professionale del singolo professionista e non dell'equipe. Le liste d'attesa sono spesso un sistema per favorire il privato fuori dall'ospedale: si comprino le prestazioni DENTRO agli ospedali e, poi, vediamo chi ha gestito meglio o peggio. Anche negli USA, regno del privato, tale tipo di attività non sarebbe tollerato (27).

Cosa ne sarebbe dell'attività convenzionata esterna? Ebbene, se la gente volesse andare verso una struttura privata convenzionata magari perché il sanitario che opera lì è ritenuto il migliore, anche questo potrebbe rappresentare un indicatore di come il pubblico ha scelto il personale sanitario e di un'eventuale cattiva formazione. Se, invece, si saturasse la disponibilità, anche in questo caso la parte amministrativa potrebbe essere chiamata a rispondere non solo della cattiva programmazione ma anche della cattiva gestione e del cattivo governo delle risorse. Oggi, infatti, un DG che abbia sbagliato a fare pianta organica, che non gestisca la qualità professionale e che non dia spazio al merito né offra prestazioni sufficienti, ma che sia capace di risparmiare, viene considerato bravo! Questo sistema permetterebbe di introdurre anche un meccanismo di valutazione politica e amministrativa. Insomma, la mia proposta va incontro ai meno abbienti e verso la qualificazione dei medici, la meritocrazia e la valutazione anche della dirigenza. $\mathrm{E}$, anche, verso un giusto riconoscimento economico per i medici disponibili a lavorare fuori orario.

\section{Disclosures}

Financial support: No financial support was received for this submission. Conflict of interest: The authors have no conflict of interest.

\section{Bibliografia}

1. Terapia d'urto per la sanità del 2016: Tre ASL, meno attese e più privati. La Nazione, articolo del 31 Dicembre 2015. http:// www.lanazione.it/riforma-sanit\%C3\%A0-toscana-1.1610316.
2. Value-Based Purchasing. National Programs to Move from Volume to Value. Articolo on-line del 26 Luglio 2012. http://www. nejm.org/doi/full/10.1056/NEJMp1204939.

3. Come mai esistono delle lunghe liste d'attesa nel servizio sanitario pubblico?. Video YouTube. https://www.youtube.com/wa tch?v=LnYuAp9wFjo\&feature=youtu.be.

4. Radiologi a congresso. Intervista a Bibbolino (Fassid Area Snr): Si prescrivono più di $100 \mathrm{mln}$ di esami all'anno ma la metà è inappropriata. Uno spreco da $500 \mathrm{mln}$ di euro. Quotidiano Sanità, articolo del 21 Maggio 2015. http://www.quotidianosanita. it/lavoro-e-professioni/articolo.php?articolo_id=28340.

5. Piano Nazionale della Cronicità. Documento della Direzione Generale della Programmazione Sanitaria del Ministero della Salute. http://www.sanita24.ilsole24ore.com/pdf2010/Editrice/ ILSOLE24ORE/QUOTIDIANO_SANITA/Online/_Oggetti_Correlati/ Documenti/2016/05/18/PIANO_CRONICITA.pdf?uuid=ADS5DwJ.

6. Documento di indirizzo per la malattia renale cronica». http://www.salute.gov.it/imgs/C_17_pubblicazioni_2244_allegato.pdf.

7. Rimandiamoal seguente articolo on-line: https://renalgate.wordpress.com/2015/06/12/global-regional-and-national-incidenceprevalence-and-years-lived-with-disability-for-301-acute-andchronic-diseases-and-injuries-in-188-countries-1990-2013-asystematic-analysis-for-the/.

8. Alfio Stiro. Agenas: nei nostri ospedali mancano medici e infermieri, ma abbondano gli amministrativi. Articolo on-line del 18 Ottobre 2015. http://www.nursenews.eu/2015/10/18/ agenas-nei-nostri-ospedali-mancano-medici-e-infermieri-maabbondano-gli-amministrativi/.

9. Cristiana Cenci. Philips crea un hub digitale per la co-produzione della salute. Articolo del 04 Gennaio 2016. http:// cristinacenci.nova100.ilsole24ore.com/2016/01/04/philipsimmagina-un-hub-digitale-per-la-co-produzione-della-salute/.

10. https://ssl.medikey.it/login_custom/login_doctor33.aspx?id= $1 \& \mathrm{t}=643$ dae5e-17d2-4357-9205-c9ee8b08efa4.

11. Cieszanowski A, et al. Non-Contrast-Enhanced Whole-Body Magnetic Resonance Imaging in the General Population: The Incidence of Abnormal Findings in Patients 50 Years Old and Younger Compared to Older Subjects. PLoS One. 2014 Sep 26;9(9):e107840. http://www.ncbi.nlm.nih.gov/ pubmed/25259581.

12. Su questo argomento specifico rimandiamo a un'intervista a Nino Cartabellotta sul sito rai news.it. http://www.rainews.it/dl/rainews/ articoli/Diagnosi-precoce-o-screening-cosa-serve-davvero-controil-cancro-59ae9cf8-dd10-418c-a3a9-8db2a8cdb4ed.html.

13. Gina Kolata. Doubt Is Raised Over Value of Surgery for Breast Lesion at Earliest Stage». Articolo del 20 Agosto 2015. http:// www.nytimes.com/2015/08/21/health/breast-cancer-ductalcarcinoma-in-situ-study.html?smid=tw-share\&_r=3.

14. Fiona Godlee. Breast screening controversy continue. BMJ 2013; 346 doi: http://dx.doi.org/10.1136/bmj.f477.

15. Grafico estratto da https://dl.dropboxusercontent.com/u/ 27140232/breqast_12003901_10154603485718521_725255 1877257974433_n.png.

16. Vinay Prasad. Why cancer screening has never been shown to "save lives"--and what we can do about it. BMJ 2016; 352 doi: http://dx.doi.org/10.1136/bmj.h6080.

17. Giuseppe Remuzzi. Perché dico sì ai tagli sanitari. Corriere della Sera, articolo del 03 Agosto 2015. http://www.marionegri.it/ media/sezione_media/Istituto_stampa/Ist_stampa_2015/GR_ tagli_sanit.pdf.

18. Curare la sanità delle liste d'attesa. II Messaggero, Aprile 2016. http://93.104.210.249/userfiles/garattini_21\%20aprile\%20 2012_def.pdf.

19. Risposta a Grattini. II Messaggero, Aprile 2012. http://www. 
anaao.it/userfiles/Risposta\%20a\%20Garattini_Messaggero_27 aprile2012.pdf.

20. Enrico Rossi Libera professione intramoenia da abolire: è fonte di disuguaglianza e corruzione. Sanità24 - 27/03/2016, II Sole 24 Ore. http://www.sanita24.ilsole24ore.com/art/ notizie-flash/2016-03-17/enrico-rossi-facebook-libera-professione-intramoenia-abolire-e-fonte-disuguaglianza-e-corruzione-110734.php?uuid=ACorKrpC.

21. Intramoenia. In campo anche Lorenzin: va regolamentata, non abolita. Replica Rossi: resto della mia idea: abolirla è d'obbligo per dignità e credibilità del Servizio Sanitario. Quotidiano Sanità, articolo del 18/03/2016.

22. Ivan Cavicchi Rossi e l'intramoenia. Lorenzin e il SSN universale. Due belle battaglie. Ma vincerle non sarà facile. Quotidiano Sanità, articolo del 21 Marzo 2016.

23. Intramoenia. Cozza (Cgil Medici): abolirla oggi rischia di essere un boomerang. Il vero allarme è il boom dei primari in extramoenia.
Quotidiano Sanità, articolo del 25 Marzo 2016.

24. John Dean, Private practice is unethical--and doctors should give it up. BMJ 2015;350:h2299.

25. Ge Bai e Gerard F. Anderson. For-profit hospitals lead the way in price gouging Extreme Markup: The Fifty US Hospitals With The Highest Charge-To-Cost Ratios Health Affairs. Giugno 2015. http://www.pnhp.org/news/2015/june/for-profit-hospitalslead-the-way-in-price-gouging.

26. G. Remuzzi. Ma la disparità tra malati c'è. Medici in fuga? Ci sono i giovani. Corriere Fiorentino, articolo del 21 Marzo 2016. http:// corrierefiorentino.corriere.it/firenze/notizie/editoriali_e_opinioni/16_marzo_20/ma-disparita-malati-c-140ea940-ee87-11e58270-ca43d80e5612.shtml.

27. Gregorio Maldini. L'intramoenia vista dagli USA. Ecco perché Rossi ha ragione. Quotidiano Sanità, articolo del 25 Marzo 2016. http://www.quotidianosanita.it/lettere-al-direttore/articolo.php?articolo_id=37962. 